\title{
Further development of the 12-item EDE- QS: identifying a cut-off for screening purposes
}

\author{
Katarina Prnjak ${ }^{1 *}$, Deborah Mitchison ${ }^{1,2}$, Scott Griffiths ${ }^{3}$, Jonathan Mond ${ }^{1,4}$, Nicole Gideon ${ }^{5}$, Lucy Serpell ${ }^{6,7}$ and \\ Phillipa Hay ${ }^{1,8}$
}

\begin{abstract}
Background: The Eating Disorder Examination - Questionnaire Short (EDE-QS) was developed as a 12-item version of the Eating Disorder Examination Questionnaire (EDE-Q) with a 4-point response scale that assesses eating disorder (ED) symptoms over the preceding 7 days. It has demonstrated good psychometric properties at initial testing. The purpose of this brief report is to determine a threshold score that could be used in screening for probable ED cases in community settings.
\end{abstract}

Methods: Data collected from Gideon et al. (2016) were re-analyzed. In their study, 559 participants (80.86\% female; 9.66\% self-reported ED diagnosis) completed the EDE-Q, EDE-QS, SCOFF, and Clinical Impairment Assessment (CIA). Discriminatory power was compared between ED instruments using receiver operating characteristic (ROC) curve analyses.

Results: A score of 15 emerged as the threshold that ensured the best trade-off between sensitivity (.83) and specificity (.85), and good positive predictive value (.37) for the EDE-QS, with discriminatory power comparable to other ED instruments.

Conclusion: The EDE-QS appears to be an instrument with good discriminatory power that could be used for ED screening purposes.

Keywords: EDE-QS, Eating disorders, Screening, ROC analysis, Discriminant validity

\section{Background}

The Eating Disorder Examination Questionnaire (EDE-Q [1];), derived from the Eating Disorder Examination (EDE [2];), is one of the most widely used and extensively validated self-report instruments for eating disorder (ED) assessment. However, the lengthy administration time of the EDE-Q and the 28-day period over which it captures symptoms may be problematic when monitoring sessionby-session outcomes in the context of clinical practice and treatment. Consequently, researchers have developed

\footnotetext{
* Correspondence: katarinaprnjak@gmail.com

${ }^{1}$ School of Medicine, Western Sydney University, Sydney, Australia

Full list of author information is available at the end of the article
}

modified versions of this tool [3]. One such version is the 12-item EDE-QS, which was recently developed employing Rasch's analysis among individuals with EDs receiving specialist treatment [4]. The EDE-QS removes the openended responses to behavioural items and also narrows the reference timeframe from the past 28-days to the past 7 -days. Alongside its brevity, this timeframe was adopted to improve recall potential, and positions the EDE-QS as a routine outcome-monitoring instrument for individuals in treatment to aid clinical decision-making via improved and regular feedback [5].

The EDE-QS has demonstrated good internal consistency, test-retest reliability, convergent validity

(C) The Author(s). 2020 Open Access This article is licensed under a Creative Commons Attribution 4.0 International License, which permits use, sharing, adaptation, distribution and reproduction in any medium or format, as long as you give appropriate credit to the original author(s) and the source, provide a link to the Creative Commons licence, and indicate if changes were made. The images or other third party material in this article are included in the article's Creative Commons licence, unless indicated otherwise in a credit line to the material. If material is not included in the article's Creative Commons licence and your intended use is not permitted by statutory regulation or exceeds the permitted use, you will need to obtain permission directly from the copyright holder. To view a copy of this licence, visit http://creativecommons.org/licenses/by/4.0/ The Creative Commons Public Domain Dedication waiver (http://creativecommons.org/publicdomain/zero/1.0/) applies to the data made available in this article, unless otherwise stated in a credit line to the data. 
and sensitivity in a mixed sample of individuals with probable ED and individuals probably not having an ED, recruited from a university and a charity [4]. Its brevity and psychometric properties provide preliminary support for the use of the EDE-QS as a screening tool for people with EDs in community settings. However, a threshold value was not established for the EDE-QS questionnaire, as it was not originally developed as a screening instrument. Cut-off scores are important for facilitating the detection of individuals likely to be experiencing threshold EDs and differentiating these individuals from those who are unlikely to have ED symptoms. Existing instruments (such as the original EDE-Q) are either too long to be practical to use for screening purposes, do not capture each disorder across the ED spectrum (for instance, SCOFF does not assess BED symptoms), or have inadequate psychometric properties in some populations (e.g. SCOFF in overweight women) [6]. Therefore, the aim of this brief report was to establish and evaluate a cutoff point on the EDE-QS that could be utilized by researchers and clinicians when identifying probable ED cases in community settings.

\section{Method}

\section{Participants and procedures}

A total of 559 people, who were recruited from a university and through a charity that offers support for eating disorders, participated in the EDE-QS validation part of the study first reported by Gideon et al. [4]. The same data are used for the secondary analysis reported in the current study. The majority of participants were women (80.86\%) aged between 18 and 34 years (92.31\%). Seventy-eight percent of the participants identified as White and $88.90 \%$ had tertiary (post-secondary school) education. Fifty-four (9.66\%) participants self-reported currently having an ED diagnosis (i.e. they responded with Yes to the question "Do you currently suffer from an eating disorder (anorexia nervosa, bulimia nervosa, binge eating disorder, eating disorder not otherwise specified)?"). Of these, 7 (13\%) met criteria for restrictive anorexia nervosa (AN-R); two (4\%) for binge eating/purging AN subtype (AN-BP); eight (15\%) for bulimia nervosa (BN); 10 (19\%) for binge eating disorder (BED); and $27(50 \%)$ for other specified feeding and eating disorders (OSFED) based on their responses on the EDE-Q and their Body Mass Index (BMI). Mean BMI in the total sample was $22.35(S D=4.61)$, with $12 \%$ of participants classified as underweight, $72 \%$ as average-weight, $12 \%$ as overweight, and $3 \%$ as obese. Underweight individuals were not excluded from the subsample of participants who self-reported currently not having an ED diagnosis. Detailed information about recruitment processes and data collection have been presented elsewhere [4]. In brief, an invitation to participate was distributed to a large university in London, UK, and the study was advertised via the Beat - Beating Eating Disorders website, a UK's eating disorder charity that offers support to people with current or former ED difficulties and their families. Participants provided informed consent and completed an online survey.

\section{Measures \\ Eating disorder examination-questionnaire short}

The EDE-QS was developed by Gideon et al. [4] as a 12item version of the EDE-Q (see below) with a response scale ranging from 0 to 3 , that captures essential symptoms of AN, BN and BED. The response scale was shortened during the development of the EDE-QS to reduce the cognitive demand and because respondents were not making full use of the 0-6 scale (some original categories were not used consistently with respondents' ED severity), as observed in Rasch analysis results [see 4]. Scores of items are summed, ranging from 0 to 36 and higher scores indicate greater ED symptoms. ED symptoms are reported for the preceding seven days. Cronbach's alpha obtained in this sample was .91 [4] indicating excellent internal consistency.

\section{Ede-q}

The most recent version of the full EDE-Q [7] is a 28item measure of ED symptoms and behaviors. Scores on each of four subscales (Restraint, Eating Concerns, Shape Concerns, Weight Concerns) and a Global score may be derived from items assessing core attitudinal features. Participants provide their answers on a scale from 0 to 6 , with higher scores indicating greater frequency and/or severity of ED psychopathology over the previous 28 days. The EDE-Q has been validated in various clinical and non-clinical samples [8]. Previous studies showed a cut-off score for "probable" ED amongst young women of 2.3 (in conjunction with the occurrence of binge eating and/or excessive exercise) [9], and a clinical cut-off of a global EDE-Q score $\geq 2.8$ [6]. In the current study sample, Cronbach's alpha was .96 for the global score and .84, .86, .93 and .88 for the Restraint, Eating Concern, Shape Concern, and Weight Concern subscales, respectively.

\section{Scoff}

The SCOFF [10] is a 5-item measure used to screen for EDs in primary care. Items tap into key symptoms of $\mathrm{AN}$ and $\mathrm{BN}$ with a dichotomous (Yes/No) response scale. The number of "Yes" responses are summed to create a total score, with a score $\geq 2$ indicative of an ED. The SCOFF has been found to have good psychometric properties in international community samples [11]. Cronbach's alpha in the current sample was .64 [4]. 


\section{Clinical impairment assessment}

The CIA (CIA 3.0) [12] was designed to measure psychosocial impairment associated with key ED features in the past 28 days. Sixteen items are answered on a 4-point Likert-type scale summed to compute the global score, with higher scores indicating greater perceived impairment. The CIA has previously been used for ED instrument validation [13,14] since clinical impairment has shown to be higher among ED clinical samples relative to healthy controls [15], thus supporting the instrument's criterion validity [16]. The CIA has robust psychometric properties [12]. In the present sample, Cronbach's alpha was .96 .

\section{Data analysis}

The R package "epiR" was used to obtain sensitivity (the proportion of true cases correctly identified by the test), specificity (the proportion of true non-cases correctly identified by the test), positive predictive values (PPV; the proportion of individuals with positive test results who have an ED), and negative predictive values (NPV; the proportion of individuals with negative test results who do not have an ED). The PPVs and NPVs depend upon the prevalence of the disorder (e.g. if the prevalence is $<10 \%$ then PPV can be $<0.5$ ) thus there is no defined criterion for classifying PPV or NPV as "acceptable," or "good" [17]. The package "pROC" was used to compute the Area Under the Curve (AUC; the surface area under the curve which describes the relationship between sensitivity and specificity) statistic and confidence intervals (CI). AUC can obtain values from 0 to 1 , with AUC of 0.50 classified as non-informative; between 0.50 and 0.70 as less accurate; between 0.70 and 0.90 as moderately accurate; between 0.90 and 1 as highly accurate; and AUC = 1 is considered as perfect [18]. Two participants $(0.36 \%)$ with missing values in the question about current ED diagnosis were excluded from the analyses (their Global EDE-Q scores were in a 30th and 38th percentile, indicating their removal would not markedly impact analyses as they did not have extreme results in this variable). Complete data were available for all other variables. Summation scores are more sensitive to missing data than other scoring methods [19], but as there were no missing data for the EDE-QS, summation was considered to be appropriate for this study. Global cut-off scores calculated as the average of item scores are also derived and presented in Table 1 in case of a necessary use by future researchers or clinicians, although for the simplicity in the following text we limit our discussion to global summation scores.

Using the self-reported ED diagnosis variable, the number of true positive, false positive, true negative, and false negative cases were determined separately for every possible cut-off score on the EDE-QS, and for the previously established range of cut-off scores on the EDE-Q (from 1.3 to 2.9) [20] and all possible SCOFF scores (from 0 to 5) [21]. Sensitivity and specificity rates were calculated for each of the possible cut-off score in these instruments, and PPV and NPV were also obtained. For the purpose of the current study, only findings for cut-off scores that yielded the highest levels of discriminatory parameters are reported, as the aim was to detect a cut-off score with high sensitivity, specificity and PPV. In addition to this, discriminatory parameters were also derived among female participants solely $(n=452)$.

Table 1 Mean (SD) of clinical impairment, EDE-Q scores, and eating disorder behaviour for self-reported eating disorder cases ( $n=$ 54) and non-cases $(n=503)$ in the current study and normative data reported by Mond et al. (2004)

\begin{tabular}{|c|c|c|c|c|c|}
\hline & \multicolumn{2}{|l|}{ Current study } & & \multicolumn{2}{|c|}{ Normative data } \\
\hline & Self-reported ED cases $(n=54)$ & Self-reported ED non-cases ( $n=503)$ & & $E D$ cases & ED non-cases \\
\hline & Mean (SD) & Mean (SD) & $t$ & Mean (SD) & Mean (SD) \\
\hline Clinical impairment & $28.67(12.44)$ & $8.24(8.47)$ & $12.15^{* *}$ & & \\
\hline Global EDE-Q & $3.99(1.39)$ & $1.67(1.32)$ & $12.25^{* *}$ & $3.09(0.83)$ & $1.30(0.96)$ \\
\hline Restraint & $3.67(1.69)$ & $1.52(1.46)$ & $9.47^{* *}$ & $2.65(1.48)$ & $1.19(1.21)$ \\
\hline Eating concerns & $3.40(1.54)$ & $0.97(1.22)$ & $11.26^{* *}$ & $2.02(0.95)$ & $0.49(0.74)$ \\
\hline Weight concerns & $4.23(1.43)$ & $1.86(1.59)$ & $12.04^{* *}$ & $3.68(1.08)$ & $1.49(1.20)$ \\
\hline Shape concerns & $4.64(1.44)$ & $2.31(1.63)$ & $11.90^{* *}$ & $4.01(0.98)$ & $2.03(1.38)$ \\
\hline OBE & $44.4 \%$ & $12.1 \%$ & $4.63^{* *}$ & $25.0 \%$ & $2.2 \%$ \\
\hline SBE & $5.5 \%$ & $9.7 \%$ & -1.23 & $25.0 \%$ & $6.0 \%$ \\
\hline Excessive exercising $^{a}$ & $27.8 \%$ & $6.4 \%$ & $3.43^{*}$ & $58.3 \%$ & $8.2 \%$ \\
\hline
\end{tabular}




\section{Results}

\section{Comparison of ED symptom levels between study} subgroups

In the absence of assignment of "ED case" status on the basis of clinical interview, preliminary analysis was conducted to confirm the validity of the "clinical" and "nonclinical" group identification, which was based on selfreported ED diagnosis. In Table 1, two groups were compared according to clinical impairment level, EDE-Q scores, and eating disorder behavior assessed by the EDE-Q. These include objective binge eating episodes (eating unusually large amount of food and having sense of losing control over eating), subjective binge eating episodes (having sense of losing control over eating without eating unusually large amount of food), and excessive exercising as a means of controlling weight or shape. Normative data for EDE-Q scores and prevalence of eating disorder behavior were also reported in Table 1 to ease comparison with present findings. As can be seen, participants with a self-reported ED had markedly elevated levels of ED symptoms when compared with the non-ED group, levels comparable to (but higher than) those of community cases of ED among young adult women identified by means of interview assessment in previous research.

\section{Comparison between measures}

As shown in Fig. 1 and Table 2, the EDE-Q and EDEQS demonstrated good, and the SCOFF excellent, discriminatory power according to the AUC statistic (AUC > .90) [18]. The EDE-Q showed an optimal sensitivity (.80) and specificity (.83) at a cut-off score of 2.8 , where the PPV and NPV reached 33 and 97\%, respectively. Similarly, with the cut-off score of $\geq 2$, the SCOFF obtained a PPV and NPV of 34 and 99\%, respectively. The EDE-QS demonstrated an optimal sensitivity (.83) and specificity $(.85)$ at a total score cut-off of 15 . At this cut-off, the NPV was very high (98\%), as with the EDE$\mathrm{Q}$ and SCOFF, while the PPV of $37 \%$ was higher than that for the EDE-Q (33\%) or SCOFF (34\%). Similar results were observed in the female subsample, with numerical differences in statistics of approximately 0.02 (higher sensitivity and PPV, lower specificity). Also, at a cut-off score of 15 on the EDE-QS, the proportion of probable ED cases in the total sample was $21.82 \%$, which is somewhat lower than the rates yielded by the EDE-Q (23.26\%) and SCOFF (25.58\%), and closer to the proportion of participants' self-reported ED diagnosis (9.66\%).

\section{Comparison of alternative EDE-QS cut-off points}

An EDE-QS score of 13 (but not 14 or 15) was within 1 SD of the mean score among participants who did not report an ED diagnosis, indicating that scores above 13 are likely to serve as cut-off points, if guided by suggestions in previous studies [20, 22]. Also, when utilizing the formula for detection of the criterion of clinical significance [23], defined as the mid-point between mean values of non-cases and cases, the result was 13.97 . However, sensitivity was the same for cut-off points of 14 and 15 , while specificity was greater with a cut-off point of 15 . When comparing mean CIA total scores between self-reported ED cases and non-cases according to each of these proposed cut-off points, the difference

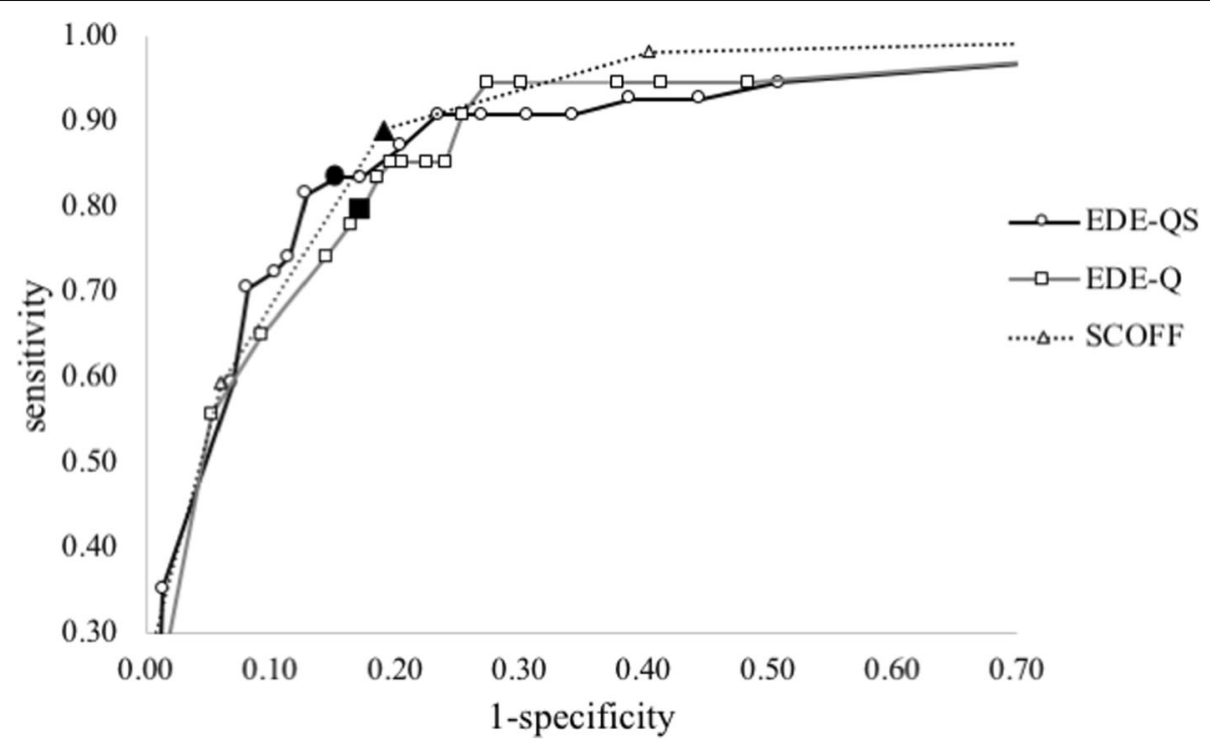

Fig. 1 Relationship between sensitivity and specificity at different cut-off scores. Black-colored markers indicate cut-off scores of 15, 2.8, and 2 for EDE-QS, EDE-Q, and SCOFF, respectively 
Table 2 Summary statistics for various global score thresholds on the EDE-Q, EDE-QS (summation/average), and SCOFF

\begin{tabular}{|c|c|c|c|c|c|c|}
\hline Cut-off & Rate of probable ED (\%) & $\operatorname{Se}(95 \% \mathrm{Cl})$ & $S p(95 \% C l)$ & $\operatorname{PPV}(95 \% C l)$ & NPV $(95 \% \mathrm{Cl})$ & $A \cup C(95 \% C l)$ \\
\hline \multicolumn{7}{|l|}{ EDE-Q } \\
\hline 2.6 & 25.94 & $.85(.73-.93)$ & $.80(.77-.84)$ & $.32(.24-.40)$ & $.98(.96-.99)$ & \multirow[t]{3}{*}{$88(.84-.93)$} \\
\hline 2.7 & 24.87 & $.83(.71-.92)$ & $.81(.78-.85)$ & $.32(.25-.41)$ & $.98(.96-.99)$ & \\
\hline 2.8 & 23.26 & $.80(.66-.89)$ & $.83(.79-.86)$ & $.33(.25-.42)$ & $.97(.95-.99)$ & \\
\hline \multicolumn{7}{|l|}{ EDE-QS } \\
\hline $13 / 1.0$ & 26.83 & $.87(.75-.95)$ & $.80(.76-.83)$ & $.31(.24-.39)$ & $.98(.96-.99)$ & \multirow[t]{4}{*}{$89(.84-.93)$} \\
\hline $14 / 1.1$ & 23.61 & $.83(.71-.92)$ & $.83(.79-.86)$ & $.34(.26-.43)$ & $.98(.96-.99)$ & \\
\hline $15 / 1.2$ & 21.82 & $.83(.71-.92)$ & $.85(.81-.88)$ & $.37(.28-.46)$ & $.98(.96-.99)$ & \\
\hline $16 / 1.3$ & 19.50 & $.81(.69-.91)$ & $.87(.84-.90)$ & $.40(.31-.50)$ & $.98(.96-.99)$ & \\
\hline \multicolumn{7}{|l|}{ SCOFF } \\
\hline 2 & 25.58 & $.89(.77-.96)$ & $.81(.77-.84)$ & $.34(.26-.42)$ & $.99(.97-.99)$ & $.90(.87-.94)$ \\
\hline
\end{tabular}

Note. ED eating disorders, Se Sensitivity, Sp Specificity, PPV Positive Predictive Value, NPV Negative Predictive Value, AUC Area Under the Curve, CI

Confidence Interval

was greatest with a cut-off point of $15\left(M_{\text {diff }}=20.05 ; 14\right.$ : $\left.M_{\text {diff }}=19.32 ; 13: M_{\text {diff }}=18.53\right)$.

\section{Discussion}

The EDE-QS demonstrated good discriminatory power, obtaining AUC, sensitivity, specificity and PPV estimates similar to those of the 28-item EDE-Q used in this study, and similar values were also reported in a study of young women in primary care [6]. Hence, reducing the number of items did not influence this instrument's ability to differentiate between self-reported ED cases and non-cases. Similar discriminatory parameters where observed between the EDE-QS and the SCOFF as well, with a higher specificity but lower sensitivity produced by the EDEQS. At the optimal cut-off point for the EDE-QS of 15, which yielded the best trade-off between sensitivity (.83) and specificity (.85) and the greatest divergence in clinical impairment between self-reported ED cases and non-cases, PPV was higher for the EDE-QS than for the SCOFF (.37 vs .34). A potential advantage of the EDEQS is that it provides more information on specific ED behaviors and their severity, which could be more clinically useful and hence outweigh the cost of the additional time taken to complete few more items. For instance, the EDE-QS captures the frequency of binge eating and purging behavior, which is important for risk and health management.

It has been suggested [21] that when establishing cutoff points for ED screening tools, priority should be given to maximizing sensitivity (i.e. lowering the criteria for reaching ED level) rather than specificity, since the purpose of these instruments is to capture potential ED cases, the status of which could then be confirmed by means of further assessment, such as a clinical interview. Prioritizing sensitivity through the selection of a slightly lower cut-off point, while conducive to an over-inclusion of non-cases, would serve to ensure the inclusion of individuals experiencing or likely to experience clinically significant impairment associated with ED symptoms and could therefore be seen as good early intervention practice [24]. The current findings suggest that a cut-off point of 15 may be optimal when using the EDE-QS for screening purposes, although a slightly lower cut-off point may be preferable if optimizing sensitivity is the goal.

\section{Limitations and directions for future research}

Some limitations should be noted. First, as in most previous studies of screening measures for $\mathrm{ED}$, men were under-represented in the current study sample. Importantly, previous research has suggested that, when compared to thresholds established for women, a lower cut-off score on ED measures may be required to identify clinically significant ED symptoms in men $[25,26]$. As the number of males with EDs in the current study was insufficient to conduct separate gender segregated analyses, further research will be needed to identify appropriate cut-off points for the EDE-QS (and other screening instruments) for men. Second, participants' age ranged between 19 and 34, calling into question the representativeness of this group for the general population, including adolescents and elderly people. Relatedly, only $15 \%$ were classified as overweight or obese which might signify the shortfall of participants who experience regular binge eating episodes. Third, ED cases were selfreported rather than independently identified which is why inferences concerning direct application of the EDE-QS as a screening instrument at this stage are necessarily tentative. Further directions for future research hence include validation in general population and primary care samples using clinical interview as a reference point. Furthermore, while the fact that the EDE-QS 
captures ED symptoms over the past 7 days may be strength in terms of facilitating participant recall, the use of this relatively short time period - far less than the period of 3 months specified in formal diagnostic criteria - underscores the point that the EDE-QS, like the EDE$\mathrm{Q}$, is not intended as and should not be used as a diagnostic instrument. Lastly, to examine the suitability of the further use of the EDE-QS as a single-factor measure of ED psychopathology, additional directions for future research include employing parallel analysis for examination of the factor structure since this method has been shown to be superior to scree-plot analysis [27], that was used in the initial EDE-QS development study [4].

\section{Conclusion}

In conclusion, as well as being a useful instrument for repeated assessments of people in treatment for an ED, the EDE-QS may be useful in screening for individuals likely to have clinically significant ED symptoms in nonclinical populations. In this study a cut-off score of 15 provided optimal validity coefficients.

\section{Abbreviations \\ ED: Eating disorders; EDE-QS: Eating Disorder Examination Questionnaire Short; EDE-Q: Eating Disorder Examination Questionnaire; AN-R: Anorexia nervosa restrictive type; AN-BP: Anorexia nervosa binge eating/purging type; BN: Bulimia nervosa; BED: Binge eating disorder; OSFED: Other specified feeding and eating disorders; BMI: Body mass index; CIA: Clinical Impairment Assessment; ROC: Receiver operating characteristic; AUC: Area under the curve; PPV: Positive predictive value; NPV: Negative predictive value}

\section{Acknowledgements}

Western Sydney University had no role in the study design, collection, analysis or interpretation of the data, writing the manuscript, or the decision to submit the paper for publication. Scott Griffiths is supported by a National Health and Medical Research Council Early Career Fellowship (grant number: 1121538) and receives honoraria from Elsevier in his capacity as Associate Editor for Body Image.

\section{Authors' contributions}

$N G, L S$ and JM conducted and wrote the original study. KP performed the analyses. KP, DM, SG, JM, NG, LS and PH wrote the paper. All authors have read and approved the manuscript.

\section{Funding}

Funding for this study was provided by the Western Sydney University as a scholarship to corresponding author (KP).

\section{Availability of data and materials}

Original data is available as a supplementary file in the manuscript published by Gideon et al. (2016): https://doi.org/10.1371/journal.pone.0152744.s004

\section{Ethics approval and consent to participate}

Ethical approval for the original data collection was obtained from a National Health Service (NHS) ethics committee (reference number: 13/EE/0254). The type of consent was "implied" since participants provided consent by proceeding with the online survey, which had been approved by the ethics committee.

\section{Consent for publication}

Not applicable.

\section{Competing interests}

Professor Hay receives/has received sessional fees and lecture fees from the Australian Medical Council, Therapeutic Guidelines publication, and New
South Wales Institute of Psychiatry and royalties/honoraria from Hogrefe and Huber, McGraw Hill Education, and Blackwell Scientific Publications, Biomed Central and PlosMedicine and she has received research grants from the NHMRC and ARC. She is Chair of the National Eating Disorders Collaboration Steering Committee in Australia (2012-) and Member of the ICD-11 Working Group for Eating Disorders (2012-2019) and was Chair Clinical Practice Guidelines Project Working Group (Eating Disorders) of RANZCP (2012-2015). In the past 5 years she has consulted for, conducted education of Psychiatrists, and prepared a report under contract for Shire Pharmaceuticals. All views in this paper are her own. Deborah Mitchison is an Editorial Board Member.

\section{Author details}

${ }^{1}$ School of Medicine, Western Sydney University, Sydney, Australia. ${ }^{2}$ Centre for Emotional Health, Department of Psychology, Macquarie University, Sydney, Australia. ${ }^{3}$ Physical Appearance Research Team, Melbourne School of Psychological Sciences, University of Melbourne, Melbourne, Australia.

${ }^{4}$ Centre for Rural Health, University of Tasmania, Launceston, TAS, Australia. ${ }^{5}$ Suffolk Family Focus Psychology Service, Norfolk and Suffolk NHS Foundation Trust, Suffolk, UK. ${ }^{6}$ Research Department of Clinical, Educational and Health Psychology, University College London, London, UK. ${ }^{7}$ North East London NHS Foundation Trust, Essex, UK. ${ }^{8}$ Camden and Campbelltown Hospitals, SWSLHD, Campbelltown, Australia.

Received: 24 January 2020 Accepted: 23 March 2020

Published online: 03 April 2020

\section{References}

1. Fairburn CG, Beglin SJ. Assessment of eating disorders: interview or selfreport questionnaire? Int J Eat Disord. 1994;16(4):363-70.

2. Fairburn CG, Cooper Z, O'Connor M. The eating disorder examination. Int J Eat Disord. 1993;6:1-8.

3. Kliem S, Mossle T, Zenger M, Strauss B, Brahler E, Hilbert A. The eating disorder examination-questionnaire 8: a brief measure of eating disorder psychopathology (EDE-Q8). Int J Eat Disord. 2016;49(6):613-6.

4. Gideon N, Hawkes N, Mond J, Saunders R, Tchanturia K, Serpell L. Development and psychometric validation of the EDE-QS, a 12 item short form of the eating disorder examination questionnaire (EDE-Q). PLoS One. 2016;11(5):e0152744

5. Simon W, Lambert MJ, Busath G, Vazquez A, Berkeljon A, Hyer K, Granley M, Berrett M. Effects of providing patient progress feedback and clinical support tools to psychotherapists in an inpatient eating disorders treatment program: a randomized controlled study. Psychother Res. 2013;23(3):287-300.

6. Mond JM, Myers TC, Crosby RD, Hay PJ, Rodgers B, Morgan JF, Lacey JH, Mitchell JE. Screening for eating disorders in primary care: EDE-Q versus SCOFF. Behav Res Ther. 2008;46(5):612-22.

7. Fairburn CG, Beglin SJ. Eating disorder examination questionnaire. Cognitive behaviour therapy and eating disorders. 2008;309:313.

8. Berg KC, Peterson CB, Frazier P, Crow SJ. Psychometric evaluation of the eating disorder examination and eating disorder examination-questionnaire: a systematic review of the literature. Int J Eat Disord. 2012;45(3):428-38.

9. Mond JM, Hay PJ, Rodgers B, Owen C, Beumont P. Validity of the eating disorder examination questionnaire (EDE-Q) in screening for eating disorders in community samples. Behav Res Ther. 2004;42:551-67.

10. Morgan JF, Reid F, Lacey JH. The SCOFF questionnaire: assessment of a new screening tool for eating disorders. Bmj. 1999;319(7223):1467-8.

11. Richter F, Strauss B, Braehler E, Adametz L, Berger U. Screening disordered eating in a representative sample of the German population: usefulness and psychometric properties of the German SCOFF questionnaire. Eat Behav. 2017;25:81-8.

12. Bohn K, Doll HA, Cooper Z, O'Connor M, Palmer RL, Fairburn CG. The measurement of impairment due to eating disorder psychopathology. Behav Res Ther. 2008:46(10):1105-10.

13. Welch E, Birgegård A, Parling T, Ghaderi A. Eating disorder examination questionnaire and clinical impairment assessment questionnaire: general population and clinical norms for young adult women in Sweden. Behav Res Ther. 2011 Feb 1:49(2):85-91.

14. Zickgraf HF, Ellis JM. Initial validation of the nine item avoidant/restrictive food intake disorder screen (NIAS): a measure of three restrictive eating patterns. Appetite. 2018 Apr 1;123:32-42. 
15. Vannucci A, Kass AE, Sinton MM, Aspen V, Weisman H, Bailey JO, Wilfley DE, Taylor CB. An examination of the clinical impairment assessment among women at high risk for eating disorder onset. Behav Res Ther. 2012 Jun 1; 50(6):407-14.

16. Jenkins PE. Psychometric validation of the clinical impairment assessment in a UK eating disorder service. Eat Behav. 2013 Apr 1;14(2):241-3.

17. Williams P, Hand D, Tarnopolsky A. The problem of screening for uncommon disorders-a comment on the eating attitudes test. Psychol Med. 1982;12:431-4.

18. Swets JA. Measuring the accuracy of diagnostic systems. Science. 1988; 240(4857):1285-93.

19. Downey RG, King CV. Missing data in Likert ratings: a comparison of replacement methods. J Gen Psych. 1998;125(2):175-91.

20. Fairburn CG, Cooper D, Phil DP. Zafra, Doll D Phil HA, O'Connor ME, Bohn D Phil DP, Kristin, hawker DM, Wales JA, Palmer RL. Transdiagnostic cognitivebehavioral therapy for patients with eating disorders: a two-site trial with 60-week follow-up. Am J Psychiatry. 2009;166(3):311-9.

21. Hill LS, Reid F, Morgan JF, Lacey JH. SCOFF, the development of an eating disorder screening questionnaire. Int J Eat Disord. 2010;43(4):344-51.

22. Rø $\varnothing$, Reas DL, Stedal K. Eating disorder examination questionnaire (EDE-Q) in Norwegian adults: discrimination between female controls and eating disorder patients. Eur Eat Disord Rev. 2015;23(5):408-12.

23. Jacobson NS, Truax P. Clinical significance: a statistical approach to defining meaningful change in psychotherapy research. J Consult Clin Psychol. 1991; 59(1):12-9.

24. Jacobi C, Abascal L, Taylor CB. Screening for eating disorders and high-risk behavior: caution. Int J Eat Disord. 2004;36(3):280-95.

25. Liu CY, Tseng MC, Chen KY, Chang CH, Liao SC, Chen HC. Sex difference in using the SCOFF questionnaire to identify eating disorder patients at a psychiatric outpatient clinic. Compr Psychiatry. 2015;57:160-6.

26. Smith KE, Mason TB, Murray SB, Griffiths S, Leonard RC, Wetterneck CT, Smith BER, Farrell NR, Riemann BC, Lavender JM. Male clinical norms and sex differences on the eating disorder inventory (EDI) and eating disorder examination questionnaire (EDE-Q). Int J Eat Disord. 2017;50(7):769-75.

27. Swami V, Barron D. Translation and validation of body image instruments: challenges, good practice guidelines, and reporting recommendations for test adaptation. Body Image. 2018;31:204-20.

\section{Publisher's Note}

Springer Nature remains neutral with regard to jurisdictional claims in published maps and institutional affiliations.

Ready to submit your research? Choose BMC and benefit from:

- fast, convenient online submission

- thorough peer review by experienced researchers in your field

- rapid publication on acceptance

- support for research data, including large and complex data types

- gold Open Access which fosters wider collaboration and increased citations

- maximum visibility for your research: over $100 \mathrm{M}$ website views per year

At $\mathrm{BMC}$, research is always in progress.

Learn more biomedcentral.com/submissions 


\section{University Library}

\section{- M M N E R VA A gateway to Melbourne's research publications}

Minerva Access is the Institutional Repository of The University of Melbourne

Author/s:

Prnjak, K;Mitchison, D;Griffiths, S;Mond, J;Gideon, N;Serpell, L;Hay, P

Title:

Further development of the 12-item EDE-QS: identifying a cut-off for screening purposes

Date:

2020-04-03

Citation:

Prnjak, K., Mitchison, D., Griffiths, S., Mond, J., Gideon, N., Serpell, L. \& Hay, P. (2020).

Further development of the 12-item EDE-QS: identifying a cut-off for screening purposes.

BMC PSYCHIATRY, 20 (1), https://doi.org/10.1186/s12888-020-02565-5.

Persistent Link:

http://hdl.handle.net/11343/246155

License:

CC BY 